\title{
AN ANALYSIS OF A THREE-PHASE VERSUS A FOUR-PHASE TRAFFIC LIGHTS REGULATION FOR A FOUR-LEG INTERSECTION
}

\author{
S.R. Pudjaprasetya ${ }^{1}$, D. Michiko M. Noor ${ }^{2}$ \\ ${ }^{1}$ Industrial \& Financial Mathematics Research Group, Faculty of \\ Mathematics and Natural Sciences, Institut Teknologi Bandung, Jalan \\ Ganesha 10 Bandung, 40514, sr_pudjap@math.itb.ac.id \\ ${ }^{2}$ Mathematics Department, Institut Teknologi Sumatera, Lampung Selatan, \\ Lampung, 35365, dear.noor@ma.itera.ac.id
}

\begin{abstract}
Traffic management of intersections is an important factor that can determine traffic density at the intersection, as well as its surrounding. Long traffic queues we encounter in daily life, were often caused by ineffectiveness of traffic lights management of the cross sections. In this article, an analytic study of traffic light management of a four-leg intersection, based on the kinematic LWR model, was presented. Comparison was based on observing the end of queues over three cycles of red-green lights, under the assumption of a constant traffic flux. On every leg of the intersection, the end of the queues were obtained from characteristic lines of the shock waves. From these observations, the three phase regulation was preferred over the four-phase one. Finally, a case study of Taman Sari - Baltos intersection located in Bandung City, Indonesia, was discussed. Parameter values used in these simulations were obtained from direct observation.

Keywords: LWR kinematic equation, shock wave, rarefaction wave.
\end{abstract}

\section{INTRODUCTION}

People living in densely populated urban areas encounter traffic congestion almost every day. When traffic volume exceeds the capacity of accessed roads, traffic congestion occurs. Heavy traffic or traffic jams encountered every day may effect our life in various negative ways. When we take a closer look, quite often traffic congestion is caused by ineffectiveness of traffic management at intersections.

2020 Mathematics Subject Classification: 65M08, 76M12, 35L65 Received: 01-11-2018, accepted: 01-04-2019. 

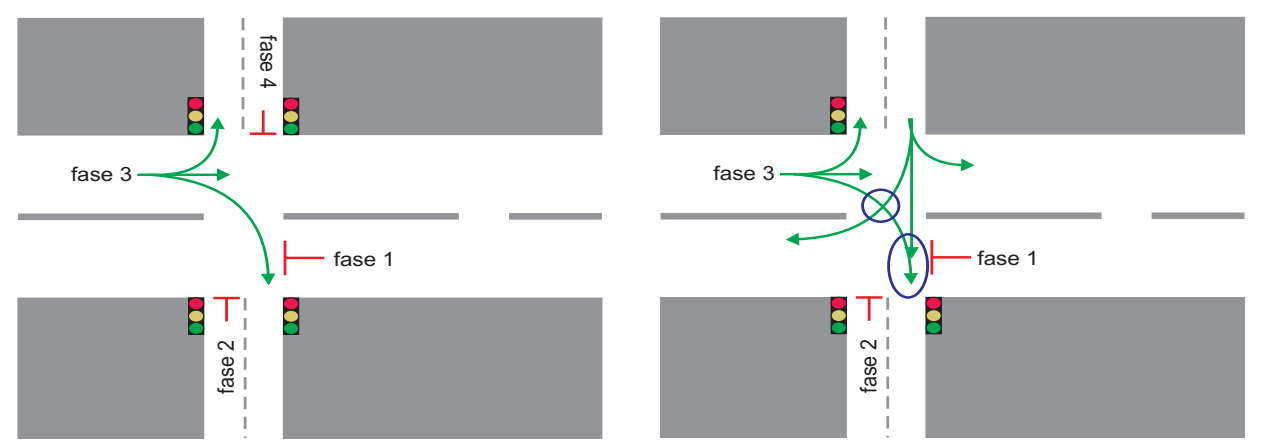

FIGURE 1. Schematic diagram of a four-leg intersection with fourphase regulation (left) and three-phase regulation (right).

At an intersection of two crossing roads, traffic lights are often used to control the traffic. Our discussion here is focused on the management of these fourleg intersections, in particular, on the comparison between a three-phase versus a four-phase traffic light regulations. Four-phase here means there will be four time intervals in one cycle period of traffic lights. As illustrated in Figure 1 (left), a four-leg intersection with a four-phase regulation, in which every leg gets its green light turn, alternately. Whereas, conducting a three-phase regulation at this cross section means there will be a phase in which vehicles from two different legs move onwards at the same time, see Figure 1 (right). Of course in the three-phase regulation, vehicles on each leg experience shorter red light interval, more or less two third of the cycle period compared to three fourth in the four-phase regulation. Hence, the three-phase traffic light regulation will be less prone to traffic jams. But three-phase regulation in a four-leg intersection may trigger conflict areas, as seen in Figure 1 (right), indicated with blue circles. In several intersections, which has a turn over, this conflict area can be avoided. Altogether we can achieved an effective traffic light regulation without conflict areas.

In this paper, we analyze the performance of the four-phase and three-phase traffic light regulations. Our approach is a macroscopic modeling of traffic dynamics using the first order kinematic equation, in which traffic movement is assumed to be a continuum. The kinematic equation is a conservative equation that has shock wave and rarefaction wave solutions. These solutions can be constructed using the method of characteristics. Detailed description about these solutions can be obtained in several literatures, for instance $[5,6]$. In [9] this kinematic model was used to compare the performance of traffic lights or a roundabout in a cross section. Another method using a second-order equations, analogous to dynamics of fluid flow has been proposed and discussed in $[1,2,10]$.

This paper is organized as follows. In Section 2, we first discuss the kinematic LWR model with Underwood flux function. Graphical representation of the shock 
wave and rarefaction wave of this equation is resumed here. In Section 3, the combination of shock wave and rarefaction wave (through their graphical representation) is used to analyze the performance of a three-phase versus a four-phase traffic light regulation in general. In Section 4, we give a case study of its application to Taman Sari - Baltos intersection. The method is implemented to assess the performance of three-phase regulation of that intersection. In this way, we can compare the performance of the four-phase and three-phase traffic regulations. Finally, concluding remarks are given in Section 5.

\section{Kinematic LWR MODEL AND THE GRAPHiCAL APPROACH}

Our approach use three important variables: traffic density $k$, average speed of vehicles $v$, and traffic flux $q$, and they are related as $q=k v$. Under the assumption that conservative principle holds, traffic flow is governed by the following kinematic Lighthill Whitham Richard (LWR) model

$$
\partial_{t} k+\partial_{x} q(k)=0 .
$$

In the macroscopic modeling of traffic flow, various drivers behavior are ignored (e.q. calm, rushed, erratic, etc.). Instead, the vehicle speed $v$ is assumed to depends only on the surrounding traffic density $k$. Once the speed function $v(k)$ is chosen, the flux function $q(k)=k v(k)$ is also obtained. Several deterministic models for $v(k)$ are as follows

$$
\begin{aligned}
\text { Greenshield } & v(k)=v_{f}\left(1-\frac{k}{k_{j}}\right), \\
\text { Greenberg } & v(k)=v_{m} \ln \left(\frac{k_{j}}{k}\right), \\
\text { Underwood } & v(k)=v_{f} \exp \left(-\frac{k}{k_{m}}\right), \\
\text { Pipes-Munjal } & v(k)=v_{f}\left(1-\left(\frac{k}{k_{j}}\right)^{n}\right),
\end{aligned}
$$

with parameters $k_{j}$ (vehicles $/ \mathrm{km}$ ) denotes the maximum traffic density or jam density, and $v_{f}(\mathrm{~km} /$ hour $)$ free flow speed. Other parameters related to maximum flux are $k_{m}$ and $v_{m}$, which denote traffic density and velocity, respectively. Detail explanation on this can be obtained in $[8,4]$. Typical value for jam density is $k_{j}=200$ vehicles $/ \mathrm{km}$, that can be obtained by assuming one lane traffic and the length of each vehicle is $5 \mathrm{~m}$. The free flow velocity parameter $v_{f}$ strongly depends on road conditions, its typical value is $v_{f}=80-100 \mathrm{~km} /$ hour for highways, and $v_{f}=40-50 \mathrm{~km} /$ hour for urban roads. In application, these parameters can be adjusted to fit field conditions. To show their relevance as traffic flow models, in $[4,13]$ these functions (2-5) were plotted against a field data measurement. Other type of speed-density relations with a discussion on parameters calibration are recorded in [13]. For a stochastic speed-density relationship, readers are referred to [12].

In Figure 2 (left) the velocity-density functions (2-5) are plotted using normalized parameters: jam density $k_{j}=1$, free-flow velocity $v_{f}=1$, parameters 


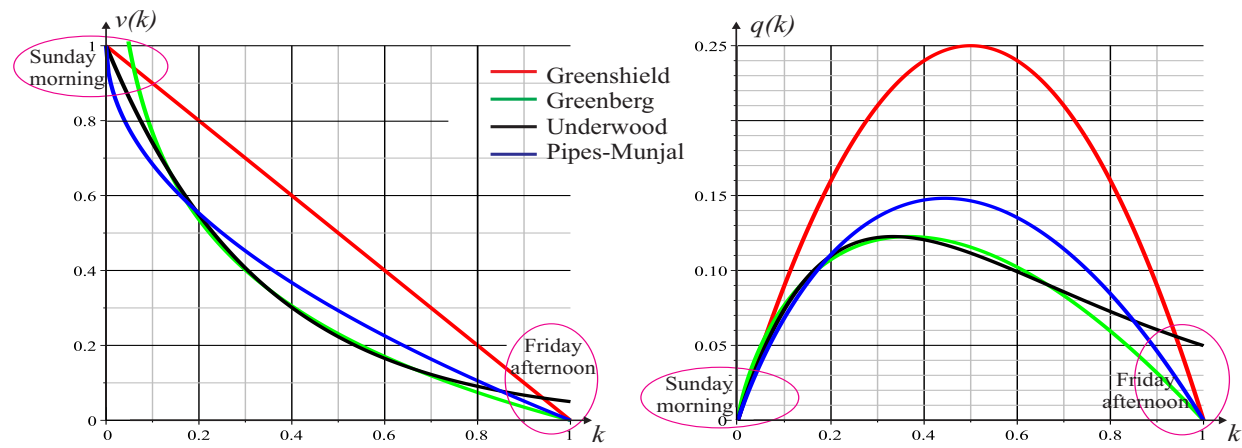

Figure 2. Several traffic flow models (left) velocity density relationship, (right) flux density relationship

related to maximum flux: traffic density $k_{m}=k_{j} / 3$, velocity $v_{m}=v_{f} / 3$, and $n=1 / 2$ for Pipes-Munjal model, whereas the corresponding flux-density functions $q(k)=k v(k)$ are plotted in Figure 2 (right). Even tough these are approximate models, they have already captured the following two extreme situations: the 'Sunday morning' situation, when there is only few vehicles on the road $k \rightarrow 0$. In this situation everyone can drive at their own speed without being blocked by other driver, so vehicles speed $v \rightarrow v_{f}$. On the other situation, typically the 'Friday afternoon' situation, when the road is jammed $k \rightarrow k_{j}$, resulting in a stop-and-go condition $v \rightarrow 0$ and traffic flux tends to zero $q \rightarrow 0$. Both situations explained above corresponds to zero limit of traffic flux. Whereas the maximum traffic flux happens for instance when traffic light turns green, in which vehicles are passing the intersection with an optimal flux.

2.1. Graphical approach. In the following, we discuss the graphical approach that will be used to assess the performance of traffic management of an intersection. To illustrate the method, let us consider a concave traffic flux curve as depicted in Figure 3 (upper left). Consider two situations where $A$ and $B$ are characterized by its flux, velocity and density: $q_{A}, v_{A}, k_{A}$, and $q_{B}, v_{B}, k_{B}$, respectively. In this example $k_{A}<k_{B}, q_{A}>q_{B}$, and $v_{A}>v_{B}$. These two conditions can be interpreted as traffic situation on highway as depicted in Figure 3 (lower left). At the outgoing part of the highway, traffic situation is dense (condition $B$ ), whereas on the incoming part, traffic situation is less dense (condition $A$ ). From top view, we observe a shock wave that connects the high density traffic $k_{B}$ to the lower density $k_{A}$. This shock wave front propagates backward with constant velocity which is the gradient $m_{A B}$ of the segment line $A B$

$$
\frac{d x}{d t}=m_{A B}=\frac{q_{B}-q_{A}}{k_{B}-k_{A}} .
$$

Velocity (6) is known as the Rangkine-Hugoniot formula. Moreover, as depicted in Figure 3 (lower left) the shock line passing through the origin determines the position of shock wave front as a function of time. This shock line also separates 
two different traffic situations: high density $k_{B}$ and low density $k_{A}$. Further, we summarize that a line segment $A B$ with negative gradient, in the flux curve, corresponds to a backwards propagating shock wave. Analogously, a line segment on the flux curve with positive gradient corresponds to a forward propagating rarefaction wave. Detailed discussion about this can be obtained in [4].

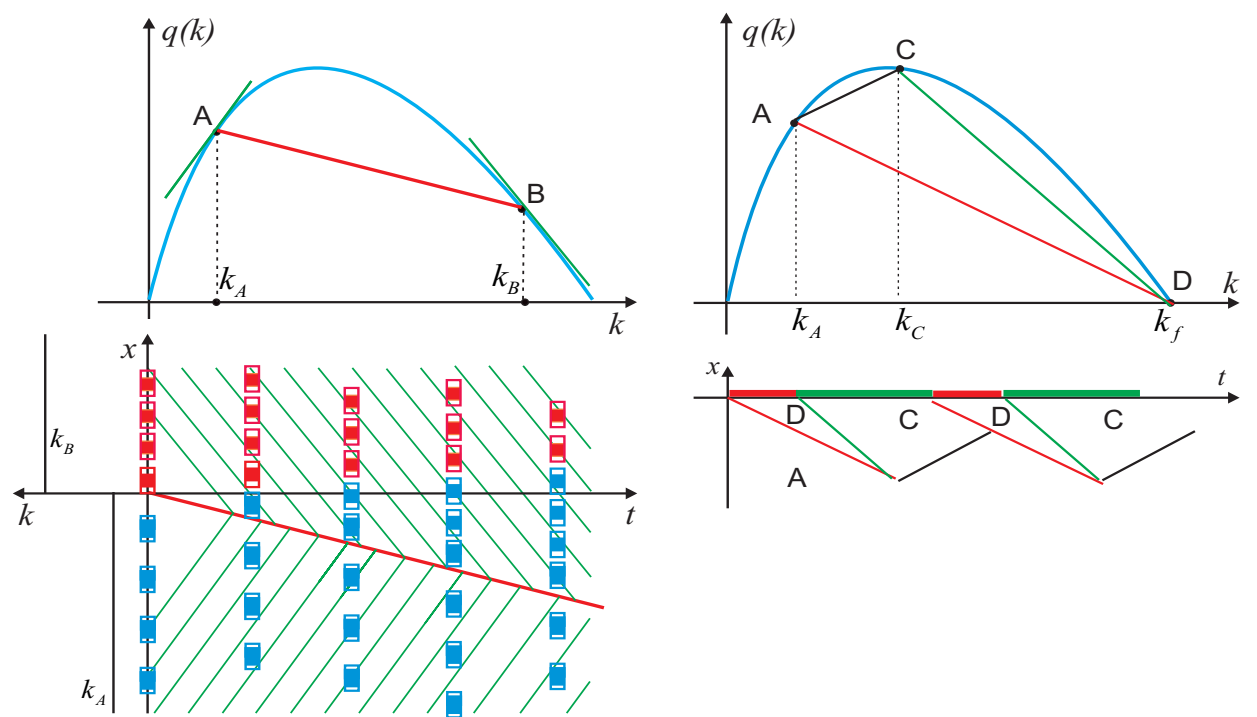

Figure 3. (Upper left) The flux curve with two traffic situations $A$ and $B$, connected with a segment line with gradient $m_{A B}$. (Lower left) Top view of a dense traffic condition $B$ propagating backward to the less dense traffic condition $A$. (Right) The flux curve with three different traffic situations A, D, C, likely happened at a traffic light. The corresponding shock lines represent transition situations as well as traffic queue.

Further, we will show how this graphical method can be used to study the effect of a traffic light. Suppose, a constant traffic density $k_{A}$ arrives at an intersection with traffic lights. Here, three different situations are involved: initial situation $A$, during red light $D$, and during green light $C$. Each of this situation is represented as a point on the flux curve in Figure 3 (right). When the traffic light turns red, vehicles queue is formed and start growing. As explained previously, the growth of this queue can be represented as a line segment with gradient $m_{A D}$ connecting the initial situation $A$ with the zero flux situation $D$. When the red light turns green, this queue of vehicles starts to release, and the situation can be expressed as a line segment $D C$, that connects the red light situation $D$ with the green light situation $C$. On the other hand, when the initial traffic meets the green 
light turn, the effect can be represented by a line segment with positive gradient $A C$.

As illustrated in the above example, any traffic situation can be represented as a point on the flux-density curve. Moreover, transition between traffic situations can also be well represented by a line segment connecting two conditions in the flux curve. This graphical method is direct and it holds for any traffic flux model in the form of a concave function. Therefore, it can be employed quite easily by traffic engineers for to analyze and manage traffic regulation.

\section{AnAlysis of a threE-Phase Versus a FOUR-PHASE}

In this section, we apply the graphical analysis to assess the performance of a three-phase versus a four-phase traffic light regulations. Actually, our analysis can take any model of speed-density relationship, and here we adopt the Underwood traffic model. This model is an exponential function, which suits best as suggested by [3]. Let us consider the following Underwood model with $k_{m}=k_{j} / 3$ or explicitly

$$
v(k)=v_{f} e^{-3 k / k_{j}},
$$

where $v_{f}$ denotes the free-flow speed, and $k_{j}$ the maximum traffic density. And the corresponding Underwood flux function is

$$
q(k)=v_{f} k e^{-3 k / k_{j}} .
$$

Here, we implement the graphical method to compare the performance of a threephase versus a four-phase traffic light regulations. For both scenarios, the cycle period is taken to be $T_{c}=60 \mathrm{sec}$. In the four-phase scenario, each leg gets the red light turn for $\frac{3}{4} T_{c}=45 \mathrm{sec}$, whereas in the three-phase regulation it is $\frac{2}{3} T_{c}=40 \mathrm{sec}$. For simulations we take normal parameters $v_{f}=1 \mathrm{~km} /$ hour, $k_{m}=1 / 3 \mathrm{veh} / \mathrm{km}$, with initial traffic density $k_{A}=0.08$. Simulations were conducted for three cycles. We first discuss the simulation of three-phase intersection, started with the red light turn, see Figure 4 (left). As time progresses, vehicles queue started to grow right behind the intersection. The growth of this queue follows the red segment line $O J_{1}$, with the end of the queue is indicated with this segment. When the light turns green, this traffic queue start to release. During this green period, the queue length decreases, indicated with the segment line $J_{1} J_{2}$. As plotted in Figure 4 (left), until the green light phase is over, some traffic queue still remains. When the next cycle starts again with the red light phase, the length of traffic queue increases again, indicated with segment $J_{2} J_{3}$. This process is repeated as time progresses.

By implementing this graphical method, we can easily track the end of traffic queue behind the intersection. Thus, we can compare the effectiveness of a three and four-phase regulation, and the one with shorter traffic queue is better. It is shown in Figure 4 that the three-phase traffic regulation gives a shorter queue, and therefore it is preferable. Intuitively, it is reasonable that the three-phase regulation is more effective because the green light phase is one third of the total 60 second of the cycle, whereas it is only one fourth of 60 second in the four-phase 


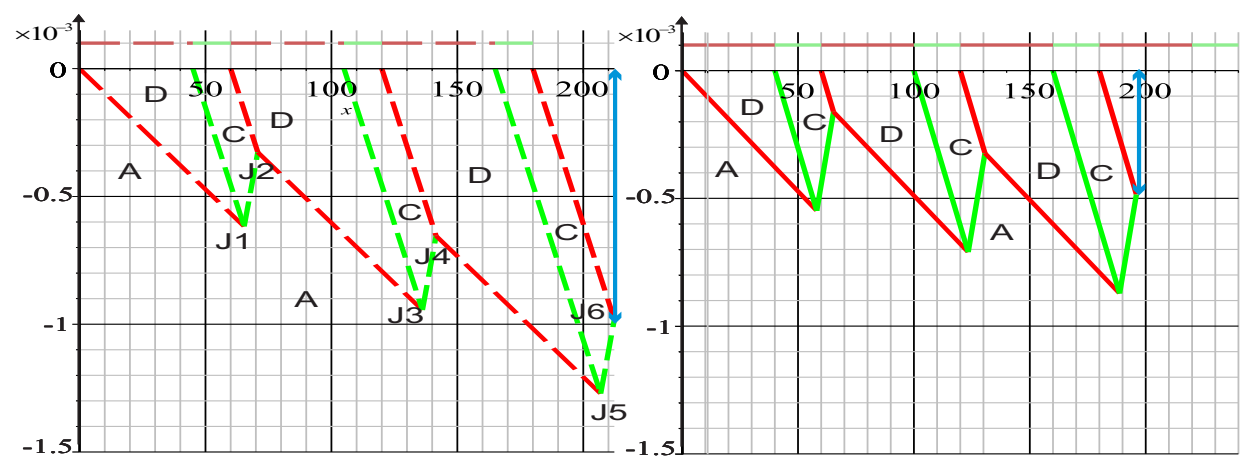

Figure 4. Characteristic lines representing traffic queue that is formed and relaxed in the four-phase regulation (left, dashed lines) versus the three-phase regulation (right, solid lines), simulated using normal parameters. The arrows indicate the length of queue after three cycle periods of traffic lights, for both regulations.

regulation. In the next section, we implement the same analysis to Taman Sari Baltos intersection at Bandung, Indonesia.

\section{A Case Study: TAman Sari - Baltos intersection}

Taman Sari - Baltos intersection is a busy intersection near Institut Teknologi Bandung campus, in Bandung, Indonesia. It is a cross section between a relatively narrow Taman Sari - Baltos street and the much wider Cikapayang - Pasupati road. Moreover, there is a flyover above Cikapayang - Pasupati road. All these roads are two-directions traffic. Along Cikapayang-Pasupati road there is a concrete structure that separates the two directions, whereas Taman Sari and Baltos do not have a road separation. Traffic light at this intersection runs under three-phase traffic regulation, with the order for the green light turns is as follows: Baltos, Pasupati, then Cikapayang. Note that in this three-phase regulation, there is no green light turn for Taman Sari. Vehicles from Taman Sari can proceed at all times, but they have to follow the U-turn route indicated in Figure 5 (left) to avoid conflict area of this three-phase regulation. Of course this triggers an increasing traffic density along Cikapayang road.

In the year 2005, this intersection applied a four-phase traffic light regulation. Is the current three-phase regulation shows an improvement of traffic management? Here, we conduct the graphical method described in Subsection 2.1 to analyze which regulation is more effective. Regulation that gives shorter traffic queue is preferable. Parameters used for the simulations are resumed in Table 1, which are based on direct measurement. It is shown that jam density for Pasupati and Baltos are $k_{j}=400 \mathrm{veh} / \mathrm{km}$, for Tamansari $k_{j}=200 \mathrm{veh} / \mathrm{km}$, Cikapayang $k_{j}=600 \mathrm{veh} / \mathrm{km}$, which is proportional to the number of lanes on these roads which are two, three, and one lane, respectively. Next, the free-flow velocity is taken to be $v_{f}=40$ 


\begin{tabular}{ccrrrrrr}
\hline Leg & phase & lane & $\begin{array}{r}k_{j} \\
(\mathrm{veh} / \mathrm{km})\end{array}$ & $\begin{array}{r}q_{A} \\
(\mathrm{veh} / \mathrm{hour})\end{array}$ & $\begin{array}{r}\text { red } \\
(\mathrm{sec})\end{array}$ & $\begin{array}{r}\text { green } \\
(\mathrm{sec})\end{array}$ & $\begin{array}{r}\text { queue } \\
(\mathrm{m})\end{array}$ \\
\hline Cikapayang & 3 & 3 & 600 & 2398 & 88 & 56 & 277.7 \\
& 4 & 2 & 600 & 1962 & 108 & 36 & 111.6 \\
\hline Pasopati & 3 & 2 & 400 & 1308 & 100 & 44 & 78.7 \\
/ Baltos & 4 & 2 & 400 & 1308 & 108 & 36 & 111.6 \\
\hline Taman Sari & 3 & - & 200 & - & - & always & - \\
& 4 & 1 & 200 & 654 & 108 & 36 & 111.6 \\
\hline
\end{tabular}

TABLE 1. Parameters used for simulation of three-phase and fourphase traffic regulations, with one cycle period of 144 sec. These parameters are based on real data collected on site.

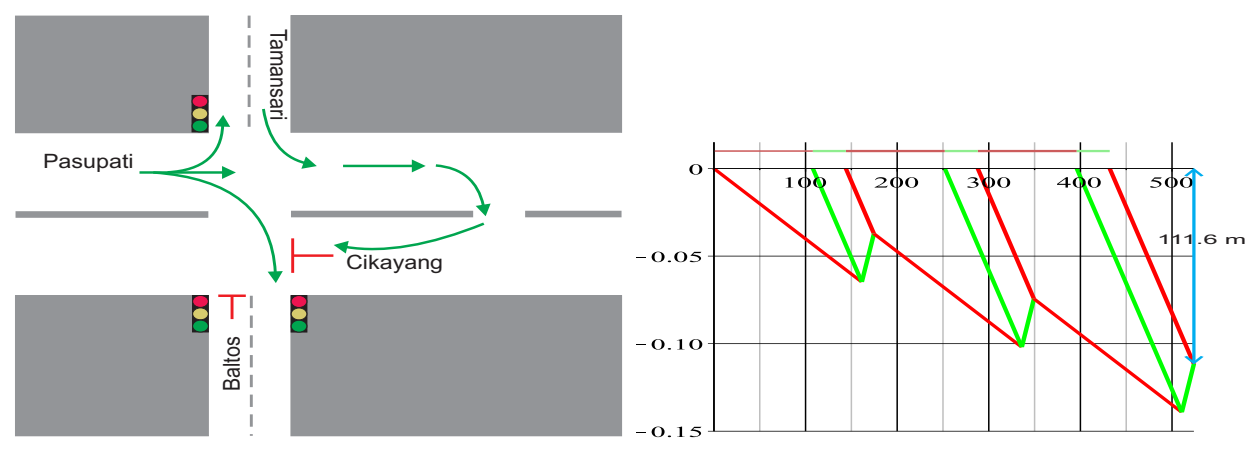

Figure 5. (Left) A schematic diagram of Taman Sari - Baltos intersection with a U-turn at Cikapayang road. (Right) Characteristic lines representing traffic queue along Taman Sari under four-phase regulation. After three cycle period of traffic lights, the simulated queue length is $111.6 \mathrm{~m}$.

$\mathrm{km} /$ hour, and one cycle period is $144 \mathrm{sec}$, with the red and green light turns for each leg (based on measurement) are given in Table 1. The initial traffic flux $q_{A}$ for each leg is taken to be the flux $q\left(\frac{2}{3} k_{m}\right)$, with $k_{m}$ is traffic density corresponds to the maximum flux. Precise value of $q_{A}$ for all legs are resumed in Table 1.

The graphical method with the Underwood flux function is used to compare those two traffic regulations. Traffic queues were formed on each leg of the intersection, and the results are depicted in Figures 5, 6, and 7. After three cycle period, the length of traffic queue for each leg is measured and recorded on the right column of Table 1 . It is clear that the three-phase regulation is effective to avoid traffic jams along Taman Sari. This is important since Taman Sari is a one lane narrow street, which easily trigger traffic congestion to its surrounding. Under this three-phase regulation, of course the other legs are affected as well. Figure 6 


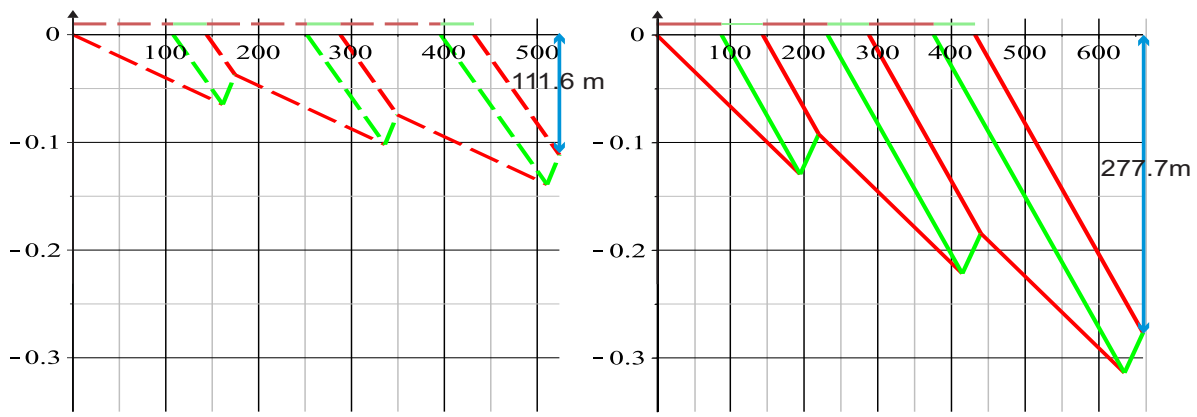

Figure 6. Characteristic lines representing traffic queue along Cikapayang. After three cycle period, the simulated queue length was $111.6 \mathrm{~m}$ under the four-phase regulation (left, dashed lines), and $277.7 \mathrm{~m}$ under the three-phase regulation (right, solid lines).

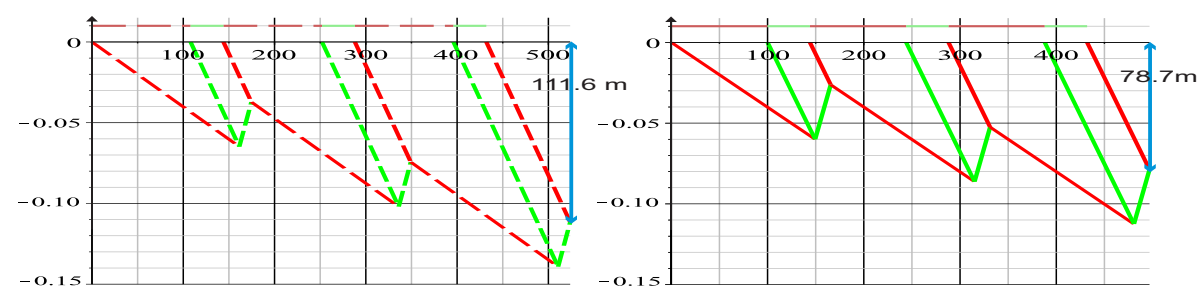

Figure 7. Characteristic lines representing traffic queue along Baltos (the same as Pasupati). After three cycle period, the simulated queue length was $111.6 \mathrm{~m}$ under the four-phase regulation (left, dashed lines), and $78.7 \mathrm{~m}$ under the three-phase regulation (right, solid lines).

shows that under the three-phase regulation, traffic queue in Cikapayang is longer compared with the four-phase regulation. In contrast, traffic queue in both Pasupati and Baltos are shorter. To sum up, the three-phase regulation shortens the length of traffic queue on three out of four legs on this intersection, which is really a good improvement of traffic management. When adopting different flux functions, all of which are concave functions, similar trend of traffic queue will be obtained, resulting in similar recommendations.

\section{Concluding Remarks}

The three-phase and four-phase traffic light regulations have been analyzed using the kinematic model with the Underwood flux function. Graphical method has been implemented, in which traffic condition during green and red lights were associated as points on the flux curve, and the growth of traffic queue behind the intersection was determined by a segment line on the flux curve. In this way, the 
length of traffic queue that was formed and relaxed during red and green lights, respectively, can be observed directly. By adopting the Underwood flux function, traffic queues that were formed in the three-phase and four-phase regulations were compared and discussed. In the simulation with normalized parameter it was shown that the three-phase regulation gave shorter traffic queue length compare with the four-phase one. Therefore, the three-phase regulation is preferable. It is important to note that in the three-phase regulation for a four-leg-intersection, any conflict area should be avoided as much as possible. This kind of policy was often implemented at several intersections in Indonesia, and our analysis provides sufficient quantitative information to encourage implementation of the three-phase traffic light regulation.

\section{REFERENCES}

[1] A. Aw, M. Rascle, Resurrection of second order models of traffic flow, it SIAM J. App. Math., Vol. 60, No. 3, (2000), 916-938

[2] P. Bagnerini, R. M. Colombo, A. Corli, S. Pedretti, Conservation Versus Balance Laws in Traffic Flow, Traffic and Granular Flow 1703. Springer, Berlin, Heidelberg (2005), 235-17240.

[3] J.M. Del Castillo, F.G. Bentez, On the functional form of the speed-density relationship I: General theory, Transpn. Res.-B, Vol. 29 No. 5, (1995), 373-17389.

[4] D. Ni, Traffic Flow Theory: Characteristics, Experimental Methods, and Numerical Techniques, Elsevier, 2015.

[5] N. D. Fowkes, J. J. Mahoney, An Introduction to Mathematical Modelling John Wiley and Sons, 1994.

[6] R. Haberman, Mathematical Models (Mechanical Vibrations, Population Dynamics, and Traffic Flow, SIAM, Texas, 1998.

[7] R.J. Salter, Highway Traffic, Analysis and Design, Springer Link, 1996.

[8] S.R. Pudjaprasetya, Transport Phenomena, Equations and Numerical Methods, ITB Press, 2018.

[9] S.R. Pudjaprasetya, J. Bunawan, C. Novtiar, Traffic light or roundabout? Analysis using the modified kinematic LWR model, E. Asian J. Appl. Math., 6 (1), (2016), 80-1788.

[10] T.Q. Tang, H.J. Huang, C.Q. Mei, S.G. Zhao, A Dynamic model for traffic network flow, Physica A 387 (11), (2008), 2603-2610.

[11] T.Q. Tang, L. Caccetta, Y.H. Wu, H.J. Huang, X.B. Yang, A macro model for traffic flow on road networks with varying road conditions, J. Adv. Transp., 48, (2014), 304-17317.

[12] H. Wang, J. Li, Q. Y. Chen and D. Ni, Speed-Density Relationship: From Deterministic to Stochastic, Transportation Research Board Annual Meeting (2009).

[13] Xu, C., Qu, Z., and Chen, X., Analysis of Traffic Flow Speed-Density Relation Model Characteristics, J. Highway Transp. Res. Dev. (English Ed.), 10.1061/JHTRCQ.0000418, (2014), 104-110. 